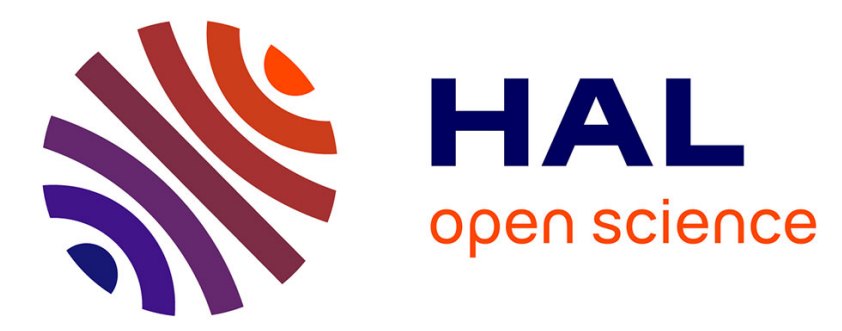

\title{
Relation entre dynamique de croissance et dynamique de prélèvement d'azote pour un peuplement de graminées fourragères. II. - Etude de la variabilité entre génotypes
} Gilles Lemaire, Jean Salette, Monique Sigogne, Jean-Pierre Terrasson

\section{- To cite this version:}

Gilles Lemaire, Jean Salette, Monique Sigogne, Jean-Pierre Terrasson. Relation entre dynamique de croissance et dynamique de prélèvement d'azote pour un peuplement de graminées fourragères. II. Etude de la variabilité entre génotypes. Agronomie, 1984, 4 (5), pp.431-436. hal-00884656

\author{
HAL Id: hal-00884656 \\ https://hal.science/hal-00884656
}

Submitted on 1 Jan 1984

HAL is a multi-disciplinary open access archive for the deposit and dissemination of scientific research documents, whether they are published or not. The documents may come from teaching and research institutions in France or abroad, or from public or private research centers.
L'archive ouverte pluridisciplinaire HAL, est destinée au dépôt et à la diffusion de documents scientifiques de niveau recherche, publiés ou non, émanant des établissements d'enseignement et de recherche français ou étrangers, des laboratoires publics ou privés. 


\title{
Relation entre dynamique de croissance et dynamique de prélèvement d'azote pour un peuplement de graminées fourragères. II. - Etude de la variabilité entre géno- types
}

\author{
Gilles LEMAIRE \& Jean SALETTE
}

avec la collaboration technique de Monique SIGOGNE \& Jean-Pierre TERRASSON

I.N.R.A., Station d'Agronomie, Route de Saint-Clément, Beaucouzé, F 49000 Angers et antenne expérimentale F 86600 Lusignan deux espèces, fétuque élevée et dactyle.

Mots clés additionnels : Fétuque élevée, dactyle, fertilisation azotée, teneur en azote, précocité, loi de dilution, variabilité interannuelle.

Additional key words : Nitrogen fertilization, nitrogen content, dilution, earliness in starting growth, tall fescue, cocksfoot, variability between years.

\section{INTRODUCTION}

Dans l'article précédent (Lemaire \& SALETTE, 1984), nous avons pu montrer qu'en conditions d'offre en azote non limitante, il existait une relation d'allométrie, stable quelle que soit l'année, entre la croissance en matière sèche et les prélèvements d'azote d'une prairie de graminées.
La grande stabilité de cette relation suggère que lorsque l'offre en azote du milieu n'est pas limitante, les prélèvements d'azote par la plante s'ajusteraient aux quantités nécessaires à la réalisation du potentiel de croissance lui-même déterminé par les conditions climatiques, notamment la température pour la période fin d'hiver - début printemps (LeMAIRE \& SALETTE, 1982). En conditions d'offre en azote 
limitée, ce serait, par contre, la vitesse de croissance qui s'ajusterait aux possibilités de prélèvement d'azote par le peuplement de graminées.

Cependant, les conditions climatiques déterminant le potentiel de croissance, notamment la température en fin d'hiver, ne sont pas sans influences sur l'offre en azote du sol (équilibre minéralisation - réorganisation) et sur la disponibilité réelle de cet azote pour la plante (développement et activité du système racinaire). Il est donc difficile d'affirmer que la relation existant entre prélèvement d'azote et croissance traduit une relation de cause à effet et que la vitesse de croissance soit le moteur unique de la dynamique des prélèvements d'azote.

Pour essayer d'approfondir cet aspect, nous avons analysé les relations entre les croissances en matière sèche et les prélèvements d'azote sur différents génotypes ayant des potentialités de croissance très différentes au début du printemps. Ainsi, nous pouvons étudier l'impact de potentialités de croissance non induites par des variations dans les conditions du milieu sur les dynamiques de prélèvement d'azote.

L'analyse de la variabilité de réponse à l'azote entre génotypes a été entreprise à Lusignan par GILLET (1972) qui a pu mettre en évidence une très forte interaction " génotype $x$ dose d'azote ». Cependant cette interaction n'apparaissait stable ni entre coupes, ni même entre années pour une même coupe. De même, un certain nombre de laboratoires de sélection des graminées fourragères ont comme objectif d'améliorer l'efficience de la réponse des génotypes à la fertilisation azotée (GOODMAN, 1977 ; ARCIONI \& LORENZETTI, 1982; SIMON, 1982).

L'étude des relations entre dynamique de croissance et dynamique de prélèvement d'azote sur une gamme de génotypes assez large nous a donc semblé intéressante pour fournir une base d'interprétation des différences de réponse constatées.

\section{MATÉRIEL ET MÉTHODES}

Le protocole: expérimental a été décrit dans le précédent article (LEMAIRE \& SAlETTE, 1984). On peut rappeler ici que la croissance des peuplements de graminées était étudiée lors de la pousse des printemps 1980,81 et 8.2 par des prélèvements successifs à la motofaucheuse.

La fumure azotée apportée, sous forme de nitrate d'ammonium, avant la pousse de chaque printemps, était de $180 \mathrm{~kg}_{\text {. ha }}{ }^{-1}$ apportés en 2 fois : $60 \mathrm{~kg}$. ha ${ }^{-1}$ après la dernière coupe d'automne, plus $120 \mathrm{~kg}$. ha ${ }^{-1}$ en fin d'hiver. Pour nous assurer que ce niveau correspondait bien à un niveau non limitant nous avions également installé des parcelles ayant reçu $60+150 \mathrm{~kg}$ d'azote. L'absence d'augmentation significative de croissance dans ces dernières nous a fait admettre le niveau $60+120$ comme non limitant.

Les génotypes étudiés étaient répartis en deux espèces :

Festuca arundinacea Schreb. cv. «Ludelle»

cv. «Clarine »

cv. « EM 77 »
Dactylis glomerata L. cv. « Lucifer»

cv. «Lubryde»

cv. "Cambria ».

Les caractéristiques de ces génotypes sont les suivantes :

« Ludelle » et «Clarine » sont des variétés de fétuque élevée de type européen. « Ludelle » a une épiaison légèrement plus tardive que "Clarine»; son départ en végétation est légèrement plus tardif. « EM 77 » est une population expérimentale obtenue à la Station d'Amélioration des Plantes fourragères de Lusignan (I.N.R.A.), issue d'un mélange de familles amphidiploïdes provenant de croisements, entre fétuque élevée de type méditerranéen et de type européen avec restauration de la fertilité par doublement des chromosomes (JADAS-HECART \& GILLET, 1978). La demi-origine méditerranéenne de cette population lui confère une précocité de départ en végétation d'autant plus importante que l'hiver est plus doux.

"Lucifer 》 est une variété de dactyle à épiaison très tardive (20-25 mai à Lusignan) et à précocité de départ en végétation également très tardive.

« Lubryde » est plus précoce à l'épiaison (10-15 mai) que "Lucifer » et de précocité comparable en départ de végétation. Sa structure génétique est celle d'un hybride de 2 lignées $I_{1}$ multipliées (MOUSSET, comm. pers.) ; il se caractérise également par une croissance très rapide lors de la montaison.

«Cambria » est une variété de dactyle sélectionnée au Pays de Galles (WPBS, Aberystwyth) à partir d'écotypes portugais. Cette origine méridionale confère à ce génotype une précocité de croissance exceptionnelle pour un dactyle en conditions d'hiver suffisamment doux.

Les conditions climatiques de 3 années 1980,81 et 82 ont été décrites dans le précédent article (LEMAIRE \& SALETTE, 1984).

On peut en résumer ici les principales caractéristiques :

1980 : fin d'hiver et début de printemps assez froid et humide,

1981 : mois de février très froid, mois de mars exceptionnellement doux,

1982 : fin d'hiver et début de printemps très doux.

\section{RÉSULTATS}

\section{A. Croissance en matière sèche}

L'analyse des courbes de croissance a déjà fait l'objet d'une publication (LEMAIRE et al., 1982). Nous avons pu montrer que la variabilité de croissance entre années pour un même génotype pouvait en premier lieu être mise en relation avec des différences de régime thermique au cours de l'hiver et en début de printemps et, à un degré moindre, avec le rayonnement reçu par la végétation en cours de croissance.

On peut constater des écarts importants dans le démarrage en croissance entre les 2 espèces lorsque les conditions thermiques de l'hiver ont permis à la 
fétuque d'extérioriser sa plus grande capacité de croissance à faibles températures. La précocité de croissance des 2 génotypes d'origine méridionale a été particulièrement marquée en 1982 en conditions d'hiver très doux et, par contre, peu nette en 1981, en conditions d'hiver froid et de réchauffement très brutal au printemps.

\section{B. Prélèvements d'azote}

Les courbes de prélèvements d'azote des différents génotypes pour chacune des 3 années sont représentées sur la figure 1 . On constate de grandes différences de prélèvements d'azote entre les génotypes en début de croissance, notamment en 1980 et 1982. Ces différences sont en grande partie expliquées par des différences de production de matière sèche.

L'analyse de ces relations entre le prélèvement d'azote et la croissance, exprimée par la matière sèche
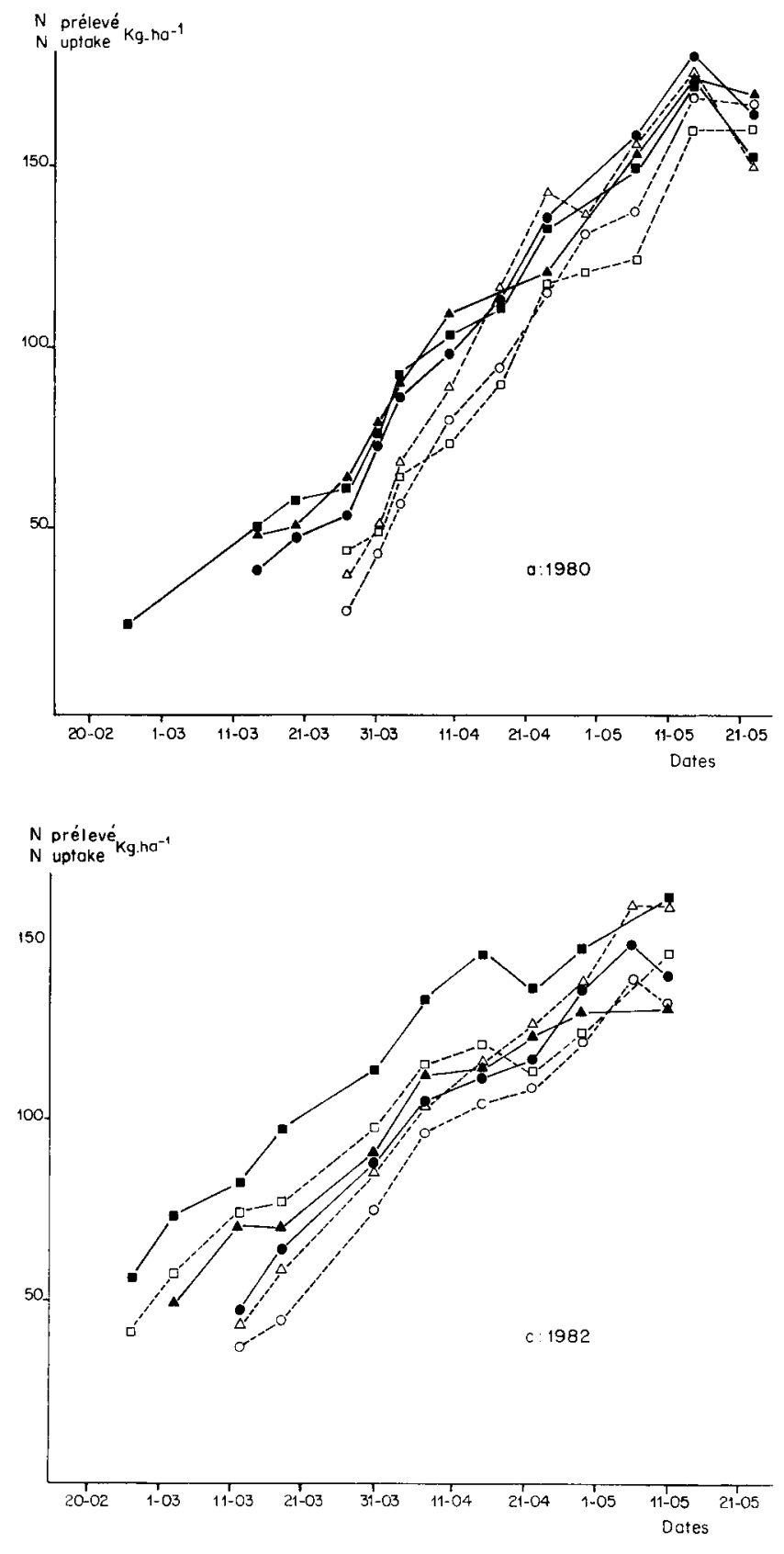

récoltable produite, en coordonnées logarithmiques (fig. 2) nous permet de déterminer des relations allométriques très étroites. Pour le début de la croissance, nous constatons qu'en 1980 et 1982 , une seule relation permet d'expliquer le comportement des 6 génotypes ; la concordance des coefficients de ces relations entre les 2 années confirme les conclusions que nous avions émises dans la note précédente. En 1981, une différence sensible apparaît entre l'ensemble des dactyles et l'ensemble des fétuques. Pour l'ensemble de ces 2 espèces, les prélèvements d'azote initiaux sont plus importants que lors des autres années : $55,7 \mathrm{~kg}$ et $64,7 \mathrm{~kg}$ d'azote pour la première tonne de matière sèche élaborée en 1981 pour les fétuques et les dactyles contre $46,2 \mathrm{~kg}$ et $51,6 \mathrm{~kg}$ respectivement pour les 2 espèces en 1980 et 1982 . Ces deux derniers chiffres sont à rapprocher des $46,7 \mathrm{~kg}$ trouvés pour la fétuque élevée «Ludelle " sur une moyenne de 6 années à Lusignan (LEMAIRE \& SALETTE, 1984).

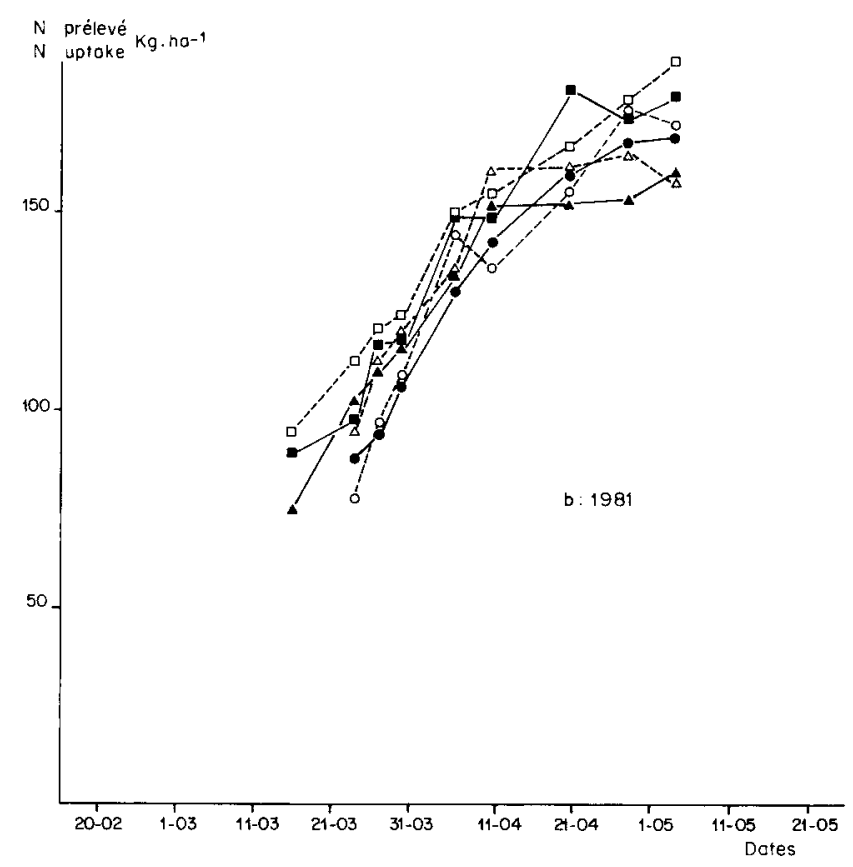

Figure 1

Prélèvements d'azote au cours de la croissance de printemps par 6 génotypes de fétuque élevée et de dactyle.

(a) : $1980 ;(b): 1981 ;(c): 1982$

fétuque élevée :

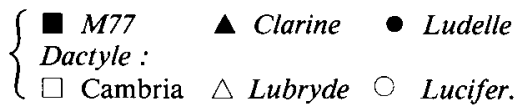

Nitrogen uptake throughout spring growth for 6 genotypes of tall fescue and cocksfoot.

(a) : $1980 ; b: 1981 ;$ (c) : 1982

Tall fescue:

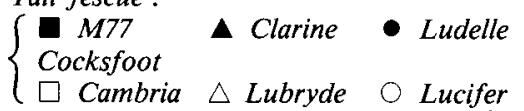



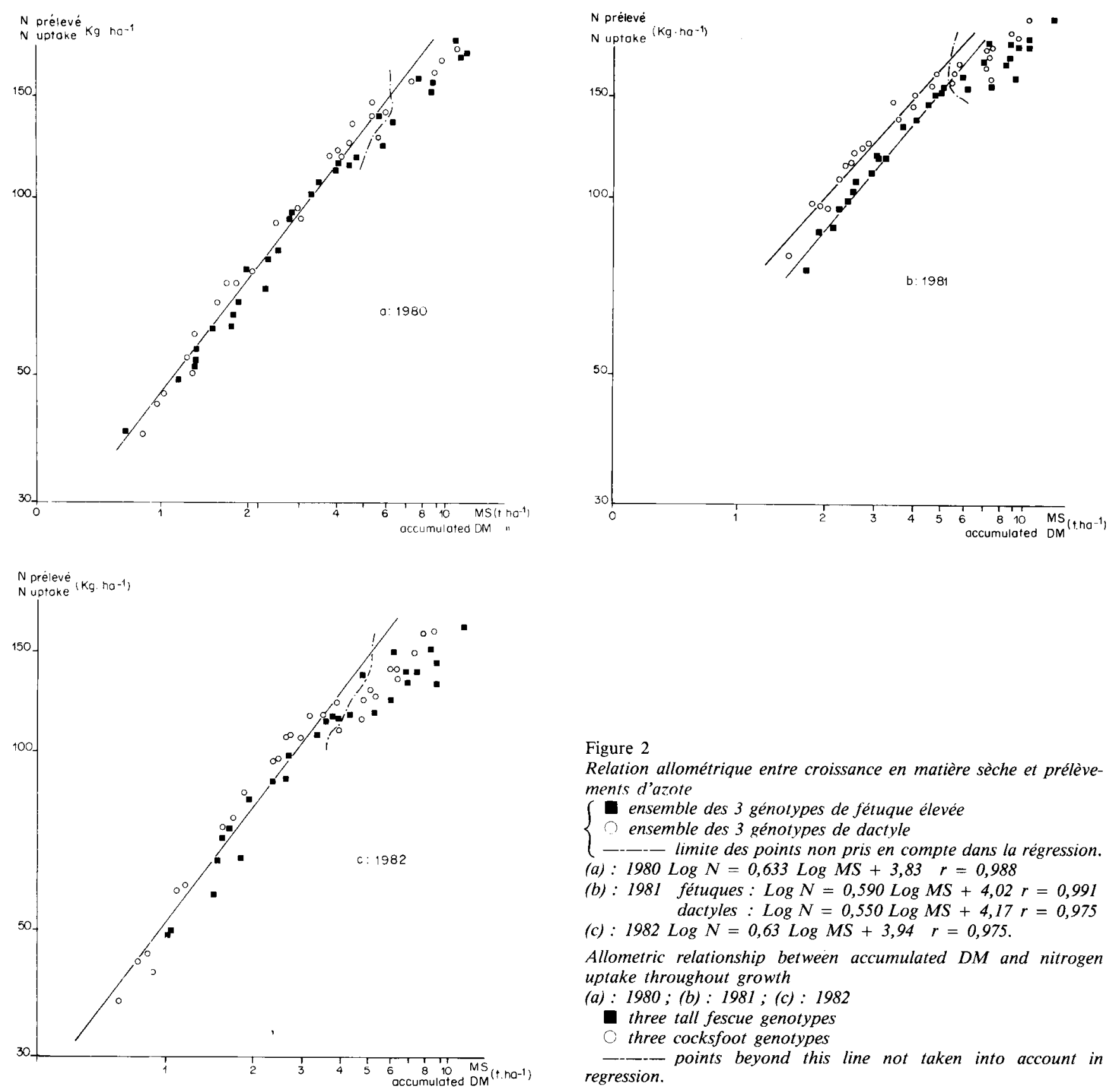

Figure 2

Relation allométrique entre croissance en matière sèche et prélèvements d'azote

$\{$ ensemble des 3 génotypes de fétuque élevée ensemble des 3 génotypes de dactyle

-_-_- limite des points non pris en compte dans la régression. (a) : $1980 \log N=0,633 \log M S+3,83 \quad r=0,988$

(b) : 1981 fétuques: $\log N=0,590 \log M S+4,02 r=0,991$ dactyles: $\log N=0,550 \log M S+4,17 r=0,975$

(c) : $1982 \log N=0,63 \log M S+3,94 \quad r=0,975$.

Allometric relationship between accumulated $D M$ and nitrogen uptake throughout growth

(a) : $1980 ;$ (b) : 1981; (c) : 1982

- three tall fescue genotypes

three cocksfoot genotypes

regression.

points beyond this line not taken into account in

nées non publiées). Dans le cas de températures du sol trop basses, cet avantage spécifique du dactyle ne se manifesterait plus.

Si l'on excepte ce comportement spécifique du dactyle, on explique l'essentiel des différences de prélèvements d'azote entre génotypes d'une même espèce par leurs différences de croissance. Encore faut-il remarquer que les différences de prélèvement entre les dactyles et les fétuques, constatées en 1981, restent faibles, de l'ordre de $10 \mathrm{~kg}$ d'azote, pour une même quantité de matière sèche produite.

Les apports d'azote intervenant simultanément sur les 6 génotypes, on peut admettre que l'offre instantanée d'azote était identique (à moins de consentir à l'existence d'une action directe du génotype sur l'équilibre minéralisation-réorganisation dans le sol). $\mathrm{La}$ concomitance presque parfaite entre prélèvements d'azote et croissance en matière sèche, lorsque la variation de cette dernière n'est plus due au milieu 
TABLEAU 1

Températures moyennes dans le sol à - $10 \mathrm{~cm}$ (Lusignan, moyennes décadaires)

Mean daily soil temperature at $10 \mathrm{~cm}$ depth (Lusignan, over ten-day periods)

\begin{tabular}{rrrrrrrrrr}
\hline & \multicolumn{3}{c}{ Février } & \multicolumn{3}{c}{ Mars } & \multicolumn{3}{c}{ Avril } \\
& 1 & 2 & 3 & 1 & 2 & 3 & 1 & 2 \\
\hline 1980 & 6,0 & 4,1 & 5,1 & 4,2 & 5,0 & 6,7 & 7,5 & 9,5 & 8,6 \\
1981 & 3,8 & 1,2 & 2,0 & 6,4 & 8,3 & 10,2 & 10,8 & 12,5 & 9,3 \\
1982 & 6,5 & 6,0 & 4,9 & 6,4 & 6,4 & 7,0 & 8,6 & 8,3 & 9,8 \\
\hline \hline
\end{tabular}

mais au génotype, amène à conclure qu'il doit exister dans la plante elle-même des mécanismes de régulation communs à ces 2 phénomènes. Cette régulation reste elle-même peu variable entre les 2 espèces, les dactyles ayant une légère tendance à augmenter plus rapidement leur prélèvement d'azote que leur croissance lorsque les conditions thermiques deviennent plus favorables.

En fin de croissance, nous observons, chaque année, une diminution des vitesses de prélèvement d'azote en fonction de la croissance. Ce phénomène étudié dans la note précédente a été interprété comme le résultat d'une diminution de l'offre en azote du milieu du fait, d'une part, des prélèvements par la plante elle-même et, d'autre part, des réorganisations plus ou moins importantes de l'azote minéral par la biomasse microbienne du sol.

Cette nouvelle observation, dans le cadre d'une comparaison entre génotypes, confirme la conclusion que nous avions faite dans l'article précédent, à savoir que le ralentissement des prélèvements en fin de croissance est bien dû à la diminution de l'offre en azote du milieu.

\section{Courbes de dilution de l'azote dans la matière sèche}

Les courbes de la figure 3, qui résultent de la relation d'allométrie illustrée dans la figure 2 et étudiée précédemment, nous permettent d'exprimer la diminution des teneurs en azote au cours de la croissance. On rend compte ainsi des corrélations négatives toujours observées, pour différents génotypes, entre la teneur en azote et la production de matière sèche. Une expression mathématique simplifiée de ces courbes peut être proposée en négligeant la rupture de pente des relations illustrées figure 2 et en déterminant une relation unique pour toute la période de croissance : les relations de la figure 3 peuvent alors être proposées comme références pour l'interprétation de données ponctuelles sur les teneurs en azote.

Ces figures peuvent être comparées à la relation plus générale illustrée par la figure 7 de la note précédente : les courbes de la figure 3 se confondent ou se situent légèrement au-dessus de la courbe de la figure 7 correspondant à l'azote non limitant. Le cas de l'année 1981, par sa courbe (fig. 3b) située audessus de la courbe de la figure 7, est un peu particulier, surtout pour le dactyle : on peut faire l'hypothèse que, pour cette année, compte tenu d'effets du climat entraînant une plus grande disponibilité de l'azote dans le sol, la dose d'azote apportée s'est située très au-delà de la dose non limitante permettant d'obtenir le maximum de matière sèche élaborée et que les teneurs plus élevées en azote observées correspondent à une consommation de luxe. Une analyse des formes d'azote soluble, notamment nitrique, eut été très instructive dans ce cas. Par ailleurs, il est intéressant de noter que le comportement des dactyles ne se différencie de celui des fétuques, au plan des courbes de dilution de l'azote, que pour des niveaux de nutrition azotée dépassant la dose qui permet d'obtenir la production maximum en matière sèche. Les teneurs en matière azotée étant, dans ces cas, inutilement élevées pour l'alimentation des ruminants, cette différence inter-spécifique est peu intéressante à utiliser.

Enfin, il est assez remarquable de constater, pour une comparaison faite à une production donnée de matière sèche, l'absence de différence de teneurs en azote entre génotypes d'une même espèce : cela indique très clairement que la seule possibilité d'augmenter par la voie génétique la production de protéines à l'hectare de prairie est de sélectionner les génotypes ayant les capacités de croissance les plus grandes.

\section{CONCLUSIONS}

La stabilité des relations allométriques entre croissance en matière sèche et prélèvements d'azote pour différents génotypes d'une même espèce confirme nos conclusions précédentes, à savoir qu'il y a réellement ajustement par la plante elle-même, à l'échelle du peuplement, des quantités d'azote prélevées dans le milieu en fonction de ses besoins propres, exprimées par son potentiel de croissance en matière sèche.

La forme des courbes indique un amortissement des prélèvements au fur et à mesure de la croissance, ce qui implique une dilution de l'azote dans la matière sèche élaborée.

Une conséquence importante de ce phénomène est que la simple comparaison de génotypes pour leur teneur en azote à un instant donné n'a aucune signification si les quantités de matière sèche produites ne sont pas prises en compte simultanément et si ces valeurs ne sont pas resituées par rapport à une courbe de dilution de référence.

$\mathrm{Au} \mathrm{vu} \mathrm{des} \mathrm{comparaisons} \mathrm{effectuées,} \mathrm{il} \mathrm{s'avère} \mathrm{que}$ la variabilité au sein d'une même espèce est très réduite et qu'elle reste faible entre les deux espèces, fétuque élevée et dactyle. 

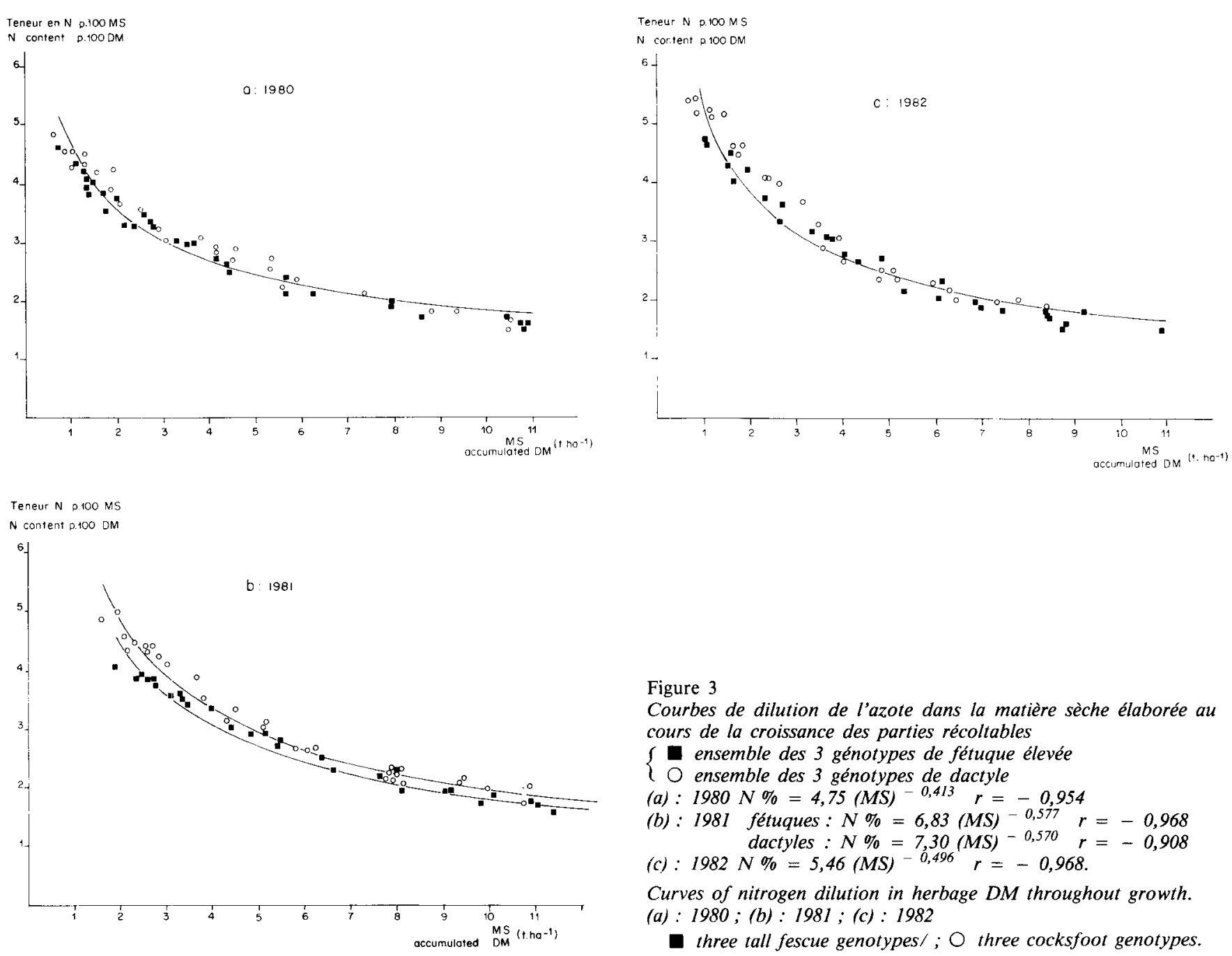

Figure 3

Courbes de dilution de l'azote dans la matière sèche élaborée au cours de la croissance des parties récoltables

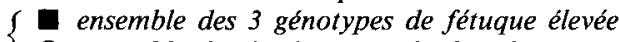

$\{$ ensemble des 3 génotypes de dactyle

(a) : $1980 \mathrm{~N} \%=4,75(\mathrm{MS}){ }^{-0,413} \quad r=-0,954$

(b) : 1981 fétuques : $N \%=6,83(M S){ }^{-0,577} r=-0,968$

dactyles : $N \%=7,30(M S)-0,570 \quad r=-0,908$

(c) : $1982 \mathrm{~N} \%=5,46(M S){ }^{-0,496} \quad r=-0,968$.

Curves of nitrogen dilution in herbage DM throughout growth. (a) : $1980 ;($ b) : $1981 ;(c): 1982$

- three tall fescue genotypes/; O three cocksfoot genotypes.

La sélection de génotypes permettant une bonne valorisation de la fumure azotée devrait reposer sur deux critères d'efficience :

Efficience de l'azote (kg MS/kg N apporté) = Efficience d'absorption ( $\mathrm{kg} \mathrm{N}$ prélevé/kg N apporté) $\times$ Efficience métabolique ( $\mathrm{kg} \mathrm{MS} / \mathrm{kg} \mathrm{N}$ prélevé) or, dans la mesure où il existe une relation étroite entre croissance et azote prélevé, très peu variable avec le génotype, la seule possibilité d'augmenter l'efficience de la fertilisation azotée et d'en économiser les apports, serait d'avoir des plantes ayant une grande efficience d'absorption, c'est-à-dire ayant la

possibilité d'extraire, malgré une offre réduite du sol, une quantité d'azote permettant une croissance suffisante. Dans cette perspective, il est également important de prendre en compte le comportement des systèmes racinaires face à deux problèmes corrélatifs importants : activité des racines dans des horizons plus ou moins bien pourvus en azote, interaction entre la rhizosphère et l'équilibre réorganisationminéralisation de l'azote dans le sol.

Reçu le 13 mai 1983. Accepté le 16 janvier 1984.

\section{RÉFÉRENCES BIBLIOGRAPHIQUES}

Arcioni S., Lorenzetti F., 1982. Evaluation of the possibility to improve protein yield in Lolium perenne. In Rep. of E.E.C. Workshop in Braunschweig, $77 \mathrm{p}$.

Gillet M., 1972. Quelques réactions de diverses graminées fourragères aux fumures azotées abondantes. C.R. Acad. Agric. Fr., 872879.

Gillet M., 1980. Les graminées fourragères. Gauthier-Villars, Paris, $306 \mathrm{p}$.

Goodman P. J., 1977. Selection for nitrogen responses in Lolium. Ann. Bot., 41, 243-256.

Jadas-Hecart J., Gillet M., 1978. Note sur les caractères agronomiques des hybrides entre fétuques élevées (Festuca arundinacea Schreb) européennes et méditerranéennes avant et après doublement. Effets de la sélection dans les amphidiploïdes. Ann. Amélior. Plant., 28 (5), 501-511.
Lemaire G., Salette J., 1982. The effects of temperature and fertilizer nitrogen on the growth of two grasses in sping. Grass Forage Sci., 37 (3), 191-198.

Lemaire G., Salette J., 1984. Relation entre dynamique de croissance et dynamique de prélèvement d'azote pour un peuplement de graminées fourragères. 1. Etude de l'effet du milieu, Agronomie, 4 (5), $423-430$

Lemaire G., Salette J., Gosse G., Chartier M., 1982. Temperature and spring growth for grasses: comparison of different grass stands. Proc. 9th Gen. Meet. Eur. Grass. Fed., Reading, 5-9 sept. 1982, ed. by Br. Grassl. Soc., 255-262.

Simon U., 1982. Efficiency of nitrogen utilisation by different types of ryegrass species. In Rep. of E.E.C. Workshop in Braunschweig, $77 \mathrm{p}$. 